Doug Geisler, Eva K. Grebel, and Dante Minniti, eds.

\title{
Old and New Tools for Understanding the Evolution of Stars in Clusters
}

\author{
Francesca D'Antona \\ Osservatorio Astronomico di Roma, I-00040 Monteporzio (Italy)
}

\begin{abstract}
The uncertainties in the modelling of some important inputs of stellar evolution must be taken into account for a correct interpretation, both of the HR diagrams of individual stars, and of the integrated colors, of stellar clusters. After a short discussion of Globular Cluster ages, we focus on the problem of convection and discuss the parametrization of convection efficiency and overshooting.

Convective efficiency affects the reliability of the giants colors, especially for high metallicity: age - metallicity relations found from integrated colors of clusters must be regarded with caution.

Non-instantaneous mixing, both in the formal convective region and in the overshooting region must be taken into account: this is necessary to compute nucleosynthesis in 'hot bottom burning' envelopes, but it may also affect the color distribution in clump (core helium burning) stars, as shown here for the case of the LMC cluster NGC 1866.
\end{abstract}

\section{Introduction}

In a Symposium dedicated mostly to the observations of Extragalactic Star Clusters it is highly necessary to go back to the basics of our knowledge of stellar evolution, to assess fairly the uncertainties in the derivation of the physically most interesting parameters of clusters, first from the star's distribution in the HR diagram, and then from the few integrated quantities we have available for the farther objects.

The study of stellar evolution is certainly one of the most advanced branches of astrophysics, and a great progress has been achieved in the latest years on all the problems of "microphysics", that is the opacities, equation of state (EOS), nuclear reaction rates and so forth. Much of this progress has been forced by the necessity of fitting properly the very detailed properties of the seismic Sun. For this purpose, e.g., the Livermore opacity project (Rogers and Iglesias 1992) was developed. Not many have noticed that the EOS built up for the computation of the Livermore opacities (Rogers, Swenson \& Iglesias 1996) has resulted to be very important for modelling both turnoff stars (Chaboyer and Kim 1995) and Horizontal Branch (HB) stars (Mazzitelli, D'Antona \& Caloi 1995, Caloi, D'Antona \& Mazzitelli 1997) in Globular Clusters (GCs), contributing to decrease the age of the most metal poor GCs, the 'stellar' age of the Universe. Nevertheless, uncertainty on this result is still quite large (section 2), as a very high degree of precision in the models is required to reduce it. 
On the other hand, there are problems in the "macrophysics" of stellar structure, whose description does not yet come from first principles, but from parametrizations based on the comparison with observations: the most important are mass loss and turbulent convection. I will discuss here this latter problem, in its different aspects (Sect. 3 and 4 ) .

\section{The age of Galactic Globular Clusters}

This is an issue in which microphysics plays a complex role. Good reviews are available (Vandenberg, Stetson and Bolte 1996, Chaboyer 2000, D'Antona 2000a), together with up to date discussions of the fits with modern stellar models (e.g. Vandenberg 2000). I wish to note here that the details of the theory determine the precise absolute ages of the galactic Globular Cluster (GC) system. The age is given by the absolute luminosity of the turnoff of the GC, so we must very precisely know the distance scale. All the methods which can be employed to derive it (main sequence (MS) fit to the subdwarfs, MS, HB and/or RR Lyrae fit to theoretical models, fit to the White Dwarf sequence or to the lower MS) end up with uncertainties $\sim 0.25 \mathrm{mag}$ in the turnoff location. As the GC age scales by $1 \mathrm{Gyr}$ for a variation of $\sim 0.07 \mathrm{mag}$ in the turnoff magnitude, the global uncertainty due to the distance scale is still at least $3 \mathrm{Gyr}$. Other uncertainties in the microphysics (e.g. the role of gravitational settling) add another $\pm 1.5 \mathrm{Gyr}$, so that the absolute age must still be considered to be $\simeq 13 \pm 3 \mathrm{Gyr}$. I add that the most recent age determinations all seem to converge towards the younger ages, and my own favourite bet is $12-14 \mathrm{Gyr}$ for the oldest GCs, but this is certainly not the final word.

\section{Convection model: its efficiency and the colors of giants}

Turbulent convection has two main aspects: the computation of the convective fluxes and the problem of chemical mixing in stars. We need a model to compute the convective fluxes. There are presently three widely adopted ways of computing superadiabatic convection in general purpose stellar evolution codes: 1) the Mixing Length Theory (MLT) by Böhm Vitense (1958); 2) the Full Spectrum of Turbulence (FST) model by Canuto \& Mazzitelli 1991; 3) the Large Eddy Simulations (LES). Limits and successes of these formulations are discussed, e.g., by Canuto \& Christensen Dalsgaard (1998), Mazzitelli (1999), D'Antona (2000b). Remember that the LES are in principle able to provide a non local description of turbulence, but the reliability of their predictions is still limited by the present computer power. The MLT and FST description both require parametric formulations for the extra-mixing or overshooting problem. It is well known, but not generally appreciated enough, that the efficiency of convection determines the stellar $T_{\text {eff }}$ for stars having important external convective layers. In the Red Giant Branch (RGB) in particular, the low densities in the envelope imply that convection is largely overadiabatic. The lower is convection efficiency, the larger is the actual temperature gradient in the overadiabatic layers, and the smaller is the resulting stellar $T_{\text {eff }}$ (or the redder are the colors). As an example, the MLT efficiency is larger the larger is the parameter $\alpha$, defined as the ratio between the mixing length and the pressure scale height. A precise value 


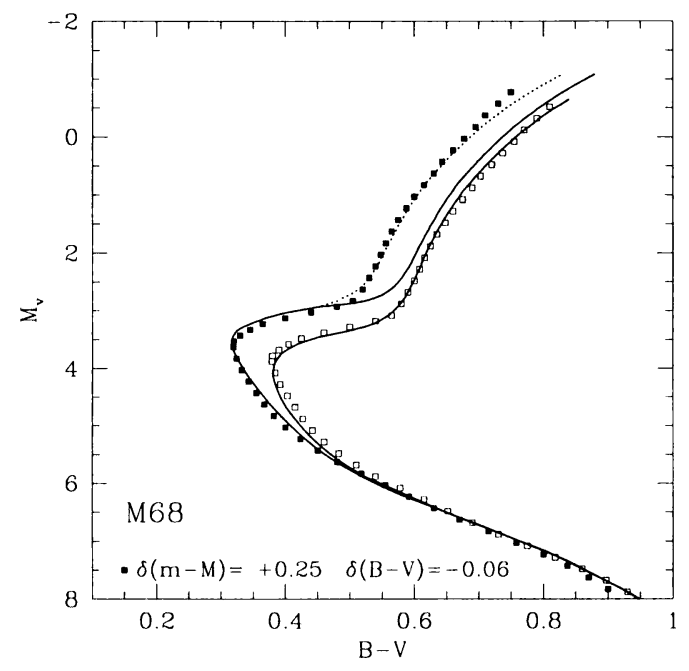

Figure 1. A schematic view of the RGB location of a metal poor GC (M68), if a 'short' distance scale (age=16Gyr open squares) or a 'long' one (age $=12 \mathrm{Gyr}$, full squares) is adopted. The RGB fit requires for the long distance scale a more efficient convection than the short one. In this sense, all set of tracks have their convection treatment 'calibrated' on the distance scale.

of this parameter can be derived by imposing the fit of the solar radius at the solar age, but there is no physical reason why convection should have the same global efficiency in stars different from the present Sun. So, the smaller is $\alpha$, the lower is the RGB $T_{\text {eff }}$. By itself, the parametrization of convection provides an uncertainty of several hundred degrees in the location of the RGB, for a given evolving mass and metallicity (Z). As we know, the RGB shifts to cooler $\mathrm{T}_{\text {eff }}$ 's by increasing $\mathrm{Z}$ (as the opacity increases), and to hotter $\mathrm{T}_{\text {eff }}$ 's by decreasing the age (as the evolving mass increases). Uncertainty in the calibration color versus $\mathrm{Z}$ may lead to uncontrolled feedbacks on our interpretation of observations. For example, we may be led to attribute a younger age to a metal rich cluster, simply as an artifact of its integrated color being bluer than the color we expect on the basis of our adopted models. For understanding the HR diagram location of single stars, and to derive information from integrated colors, we must know exceedingly well the functional dependence of the RGB location on the age and on $\mathrm{Z}$.

\subsection{The 'hot' boundary of the RGB location}

First of all, models for population synthesis must reproduce the location of the most metal poor GCs. As the convection theories presently available can not predict the RGB $T_{\text {eff }}$, our models must choose an appropriate calibration (in fact, we do not fit $T_{\text {eff' }}$ 's, but colors, so that we also need to know the correspondence between colors and $\left.T_{\text {eff }}\right)$. It is easy to convince ourselves that the location of 


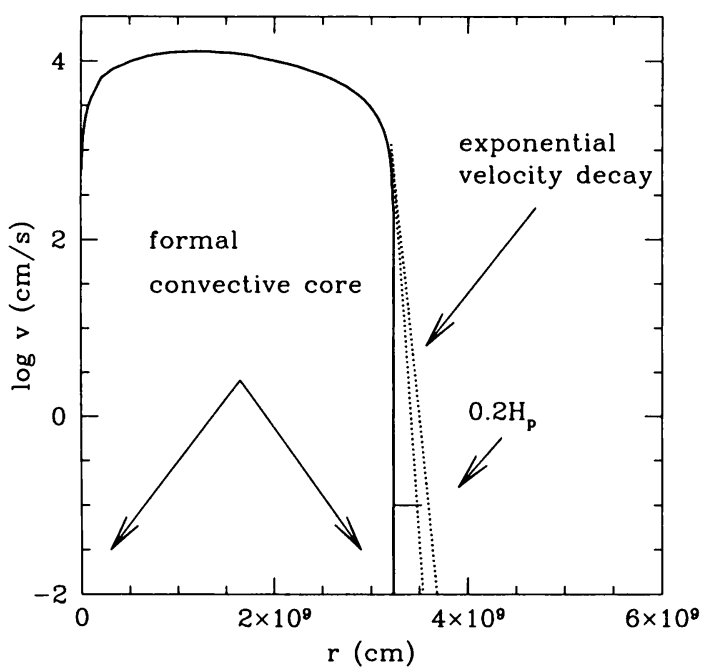

Figure 2. The average velocity inside the core of a MS $4.5 \mathrm{M}_{\odot}$ star is shown, computed according to the FST model by Canuto, Goldman and Mazzitelli 1996. The dashed lines show the extrapolation of the convective velocities assumed in the models with non instantaneous mixing and overshooting. The sharper decline corresponds to the overshooting scale $o v=0.02$, the other to $o v=0.03$.

old metal poor giants in the magnitude - color plane depends in a subtle way on the adopted distance scale (or age). Figure 1 shows in a schematic way that distance scales differing by $0.25 \mathrm{mag}$ for the GC M68 result either in a fit with $16 \mathrm{Gyr}$ (on the right, open squares) or with $12 \mathrm{Gyr}$ - after adjusting the colors of the diagram by $0.06 \mathrm{mag}$. But, if the stellar models were able to fit the giants location at an age of $16 \mathrm{Gyr}$, the same models provide a RGB too cool for the younger age. The obvious way to have again the RGB fit is to increase the efficiency of convection, e.g. by increasing the $\alpha$ in the stellar models. In this way we see that the left boundary of the old, metal poor RGBs is "fixed by the distance scale of the metal poor GCs". In the case exemplified, the uncertainty amounts to $\sim 0.18 \mathrm{mag}$ in the average $B-V$ color of the RGB. Actually, it is less severe, as there are many metal poor GCs which can be used as calibrators, opacities are relatively well known just because $\mathrm{Z}$ is very low, and it is not unrealistic to agree to the common belief that the metal poorest GCs are all coeval.

\subsection{The 'cool' boundary of the RGB location}

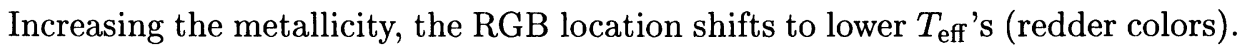
As we can not trust blindly the dependence $\mathrm{Z}-T_{\text {eff }}$ of our models, even if we have well calibrated them at low $\mathrm{Z}$, we must be able to define and make use of a 'right boundary' to the RGB location. This task is not yet well exploited, due to the following problems: 
1. Calibrators for higher metallicity become less and less secure, as the 'observational' metallicity is not certain, particularly if we have also to consider possible $\alpha$-elements enhancements. Further, an age spread is possible. Let us consider that ages from 8 to $14 \mathrm{Gyr}$ appear in the recent literature for the GC 47Tuc (Salaris \& Weiss 1998; Vazdekis et al. 2001), which certainly is not extreme in metallicity $([\mathrm{Fe} / \mathrm{H}] \sim-0.9$ with $[\alpha / F e] \sim 0.4)$.

2. The $T_{\text {eff's }}$ of the RGB become quite low: below $\sim 4000 \mathrm{~K}$, triatomic molecules (in primis $\mathrm{H}_{2} \mathrm{O}$ ) must be included in the opacity computations remember that they are not included in the widely used Kurucz's libraries-; model atmospheres must no longer rely on simple grey integration to be used as boundary conditions to the stellar models.

3. Even if we knew how to deal with convection, the computation of the overadiabatic gradient requires knowledge also of the radiative gradient: consequently, we need a detailed opacity treatment, also at large optical depth.

My conclusion is that we can not state that we know the RGB location for 'large' metallicities well enough to trust synthetic evolution models, in order to infer in detail the age of compact distant stellar systems from their integrated colors. In particular, any correlation age $-\mathrm{Z}$ derived from integrated colors must be well checked in order to understand whether it is not mainly a result of the assumed theoretical dependence of the RGB location on the metallicity.

\subsection{The 'hot bottom' of convective envelopes}

The efficiency of convection determines also the structure of a distinct group of stars: the very luminous Asymptotic Giant Branch (AGB) stars, which are known to reach, at the bottom of their convective envelope, temperatures which allow nuclear burning. There are today many models which successfully predict, e.g., the formation of Lithium in the envelopes of the luminous AGBs (Sackmann and Boothroyd 1992, Mazzitelli, D'Antona \& Ventura 1999, Blöcker, Herwig \& Driebe 2000) in agreement with their observational counterparts of the Magellanic Clouds (Smith et al. 1995). The minimum mass which suffers 'hot bottom burning' (HBB) depends on the metallicity and on the convection efficiency. A possible way to study the convection efficiency is then to search for Lithium rich AGBs in clusters of known age (and thus, in principle, of known evolving mass). Such stars have been recently found in two young clusters of the LMC: one in NGC 1866 and one in NGC 2031 (Maceroni et al. 2001).

\section{Convection model: mixing beyond the convective boundary}

A second aspect of convection in stars is the chemical mixing associated with convection, and in particular the mixing which extends beyond the formal convective boundaries, defined in most cases by the Schwarzschild criterion. This problem is generally treated in the context of an 'overshooting' problem, although there are other physical mechanisms which can result in an extension of the stellar convective cores larger than predicted by standard stellar models (e.g. rotation, Maeder \& Meynet 2000). In past years, an inadequate knowledge of opacities was at the basis of prediction of convective cores smaller than required by the observations, as shown at the introduction of OPAL opacities (Roger \& Iglesias 1992, see e.g. Stothers \& Chin 1992). I wish to discuss only 


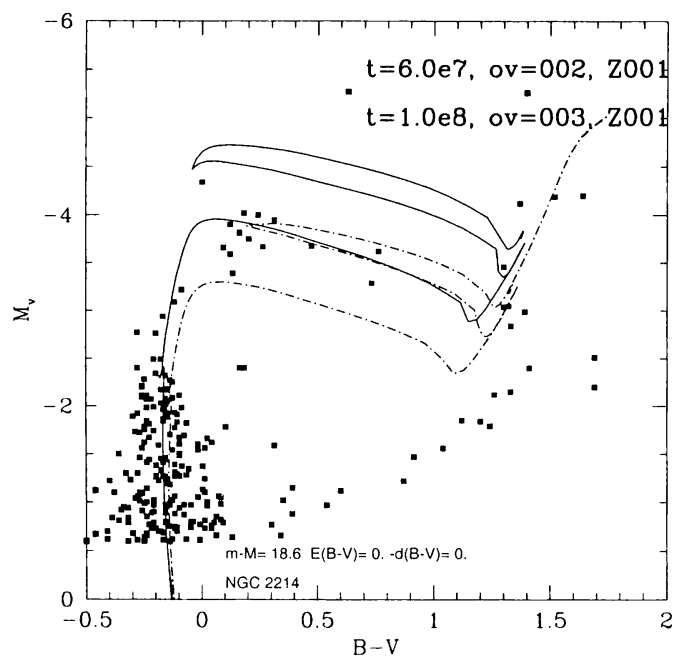

Figure 3. HR diagram of the LMC cluster NGC 2214, and two isochrone 'fits': only the older age (solid line) is realistic, based on the expected number of clump stars.

'overshooting' here, that is the extra mixing due to the fact that the convective eddies arrive at the Schwarzschild boundary with zero acceleration, but with a finite velocity which can allow them to go beyond it. A self-consistent treatment of this problem has been until now beyond solving possibilities, as a non local model for convection is necessary. The analytic approaches have been unfruitful for most cases of astrophysical interest (that is: when extended convective regions are present, but see, e.g., Kupka 1999 for the description of thin convective envelopes), and the LES can not yet deal with the description of the turbulence at all the scales present in stars. Consequently, stellar models still adopt a local description of turbulence (like the MLT or FST) and a fully parametric approach for the overshooting.

In addition, most stellar models adopt a very rough approach to describe overshooting: it is assumed that the convective mixing proceeds for a given amount beyond the formal convective border. This is generally parametrized in fractions of $H_{p}$ at the border. A value of $\sim 0.2 H_{p}$ seems plausible and is adequate to fit, e.g., the observed thickness of the main sequence. A dependence of the degree of overshoot on the stellar mass (larger overshooting the larger is the mass) seems however to be required. This enlarges the number of parameters we need to describe convection and does not allow us to make predictions. Deng et al. (1996) introduced non-instantaneous mixing assuming an exponential decay of the convective velocities outside the formal convective region. A similar approach has been introduced by Ventura et al. (1998). Figure 2 shows the convective velocities in the core of a $4.5 M_{\odot}$ star according to the FST treatment (Canuto et al. 1996). As the convection model is local, the velocity decreases to zero both at the inner and outer boundaries. The spurious decrease at $r=0$ is simply ignored in the model. At the outer boundary, we extrapolate the velocity 

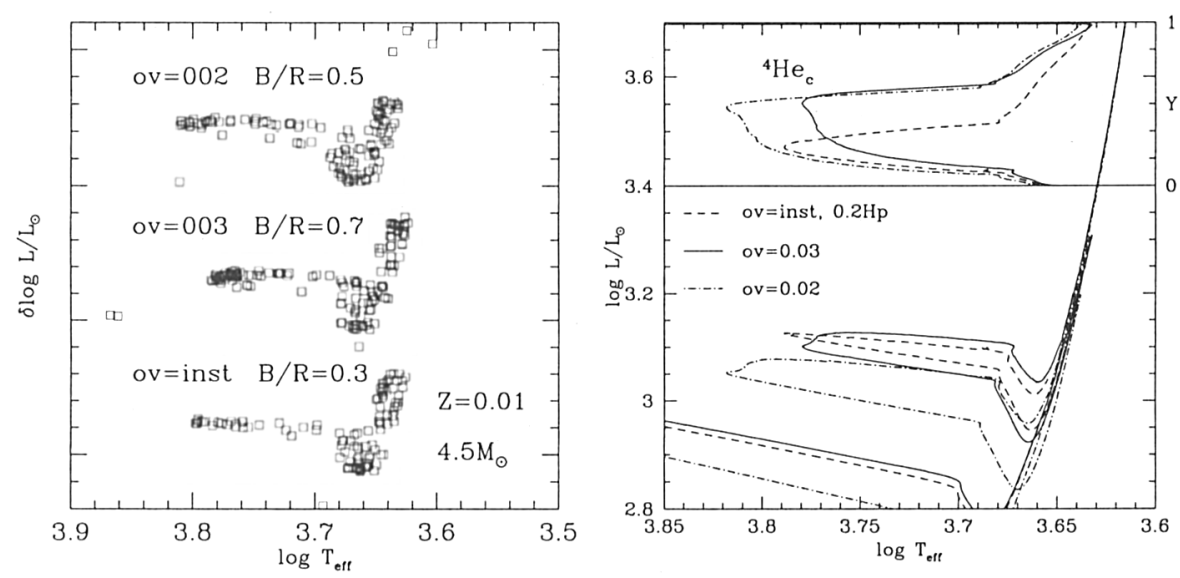

Figure 4. On the right the evolutionary tracks for $4.5 \mathrm{M}_{\odot}$ and different overshooting treatment are shown, displaying on top the core helium abundance $\mathrm{Y}$ along the evolution. The left figure shows simulations based on the tracks: the number ratio of clump stars (B) to RGB stars (R) predicted by each model is given. The observed ratio is $\sim 0.9$. The $o v=$ inst model predicts very few stars in the blue loop.

before its sharp decrease, and impose that the eddy velocities decay exponentially, with a given e-folding distance (ov) (details in Ventura et al. 1998). A $0.2 H_{p}$ overshoot is also shown for comparison. Mixing inside the convective and overshooting regions is solved, together with the nuclear evolution matrix, as a time dependent diffusion process (diffusive mixing). This modality of mixing is substantially different from the instantaneous mixing approximation, and the models must be compared to observations to understand whether they improve the agreement. This is being done by Tosi et al. (2001) with a synthetic HR diagram approach, superior to the plain comparison of isochrones: this latter in fact does not take into account the lifetimes in the different evolutionary phases. As an example, Figure 3 shows two isochrones superimposed on the HR diagram of the young cluster NGC 2214 in the LMC. For this cluster, Subramaniam \& Sagar (1995) derive an age of $40-60 \mathrm{Myr}$, but the stars which seem to be postMS when compared to the $60 \mathrm{Myr}$ isochrone, may actually be in the clump stage of a $100 \mathrm{Myr}$ isochrone, as it can be shown by comparing with synthetic HR diagrams.

\subsection{The clump of NGC 1866}

A detailed study of the core-He burning phase with diffusive mixing and overshooting will be given by Ventura et al. (in preparation). The extension and duration of the blue loop depends on the fine balancing between three main parameters: 1) the intensity and phase of core He-burning; 2) the ratio between the efficiency of the CNO H-burning shell and the core He-burning; 3) the opacity in the surface layers (Iben 1967). These parameters are influenced by the modelling of convection, both through the temperature gradient (3) and 

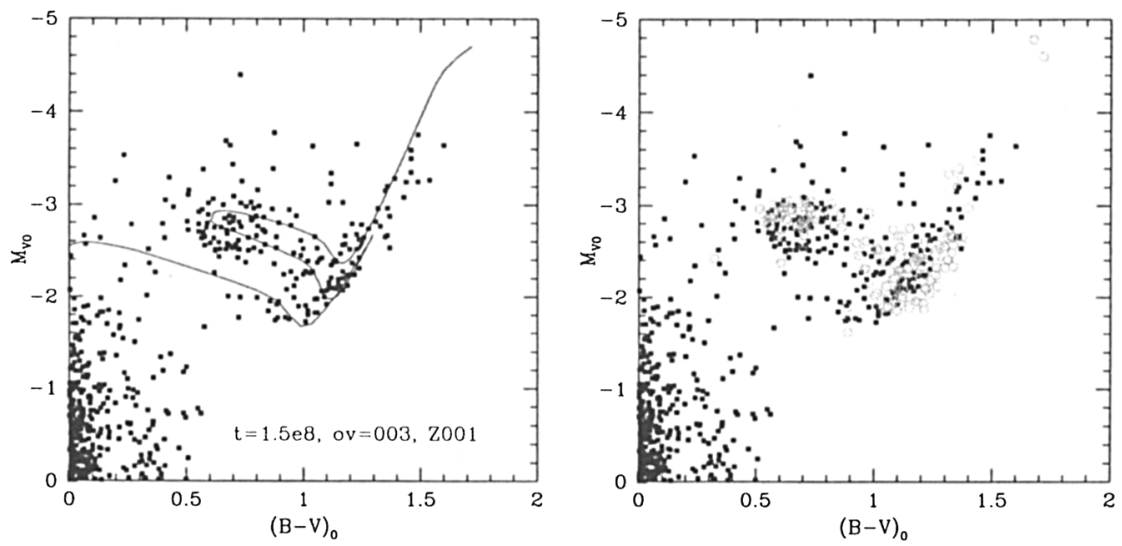

Figure 5. In the left figure, an isochrone of $150 \mathrm{Myr}$ is superimposed on the HR diagram of the clump stars of NGC 1866 (Testa et al. 1999) (distance modulus $18.6 \mathrm{mag}$ ). On the right, a simulation of the cluster population is superimposed (open circles).

through overshooting in this phase and in the preceding ones (1 and 2). We can find very fast and/or small loops, or extended and long lasting loops depending on the inputs. Figure 4 exemplifies the results obtained for different convection models in the case of a $4.5 \mathrm{M}_{\odot}$ evolution, which well describes the evolution in the cluster NGC 1866. The degree of overshooting and its modalities (diffusive versus instantaneous) determine the location of the clump and the ratio between "blue" (B) and "red" (R) stars. The instantaneous treatment of overshooting in this case provides the worst performance.

In conclusion, the HR diagram of NGC 1866, and in particular its clump, is well reproduced with the models with ov $=0.03$, for an age of about $1.5 \times 10^{8} \mathrm{yr}$. Figure 5 shows the comparison of the HR diagram by Testa et al. (1999) with the isochrone (left) and with the synthetic model (right). At this age, a mass of $\simeq 4.5 M_{\odot}$ is evolving in the AGB, and for this mass our models predict $\mathrm{HBB}$ and the production of Lithium, confirmed to be present in one AGB star of the cluster by Maceroni et al. (2001). Although still very parametric, these models seem to offer very detailed performances in the comparisons with observations, and allow us a step ahead in the understanding of stellar clusters.

Acknowledgments. I thank very much Eva Grebel and Doug Geisler for inviting me to deliver this talk and for their organizational effort (resulting in a very good Symposium and a spectacular environment). This work has been supported by the Ministero dell'Università e della Ricerca Scientifica e Tecnologica under the program: "Stellar Dynamics and Stellar Evolution in Globular Clusters". 


\section{References}

Blöcker, T., Herwig, F., Driebe, T. 2000, The Changes in Abundances in Asymptotic Giant Branch Stars, Eds. F. D'Antona \& R. Gallino, Mem.S.A.It. 71,711

Böhm-Vitense, E. 1958, Z. Astrophys., 46, 108

Caloi, V., D'Antona, F., \& Mazzitelli, I. 1997, A\&A, 320, 823

Canuto, V. M. \& Mazzitelli, I. 1991, ApJ, 370, 295

Canuto, V. M., Goldman, I., \& Mazzitelli, I. 1996, ApJ, 473, 550

Canuto, V.M., \& Christensen Dalsgaard, J. 1998, Ann.Rev. Fluid Mech., 30,167

Chaboyer, B. 2000, The Galactic Halo : From Globular Cluster to Field Stars, 35th Liege International Astrophys. Coll. Edited by A. Noels et al.. Liege, Belgium : Institut d'Astrophysique et de Geophysique, p.451

Chaboyer, B. \& Kim, Y. 1995, ApJ, 454, 767

D'Antona, F. 2000a, The Galactic Halo : From Globular Cluster to Field Stars, 35th Liege International Astroph. Coll.. Edited by A. Noels et al.. Liege, Belgium : Institut d'Astrophysique et de Geophysique, p.433

D'Antona, F. 2000b, Very low mass stars and brown dwarfs, Edited by R. Rebolo and M. R. Zapatero Osorio, Cambridge Contemporary Astrophysics, Cambridge University press, 193

Deng, L., Bressan, A., \& Chiosi, C. 1996, A\&A, 313, 145

Harris, G. L. H. \& Deupree, R. G. 1976, ApJ, 209, 402

Iben, I.Jr. 1967, ARA\&A, 5, 571

Kupka, F. 1999, ApJ, 526, L45

Maceroni, C., Testa, V., Plez, B., D’Antona, F. \& Garcia Lario, P. 2001, in preparation

Maeder, A. \& Meynet, G. 2000, ARA\&A, 38, 143

Mazzitelli, I., D'Antona, F., \& Caloi, V. 1995, A\&A, 302, 382

Mazzitelli, I. 1999, ASP Conf. Ser. 173: Stellar Structure: Theory and Test of Convective Energy Transport, 77

Mazzitelli, I., D’Antona, F., \& Ventura, P. 1999, A\&A, 348, 846

Rogers, F. J. \& Iglesias, C. A. 1992, ApJ, 401, 361

Rogers, F. J., Swenson, F. J., \& Iglesias, C. A. 1996, ApJ, 456, 902

Sackmann, I. -. \& Boothroyd, A. I. 1992, ApJ, 392, L71

Salaris, M. \& Weiss, A. 1998, A\&A, 335, 943

Smith, V. V., Plez, B., Lambert, D. L., \& Lubowich, D. A. 1995, ApJ, 441, 735

Stothers, R. B. \& Chin, C. 1992, ApJ, 390, 136

Subramaniam, A. \& Sagar, R. 1995, A\&A, 297, 695

Testa, V., Ferraro, F. R., Chieffi, A., Straniero, O., Limongi, M., \& Fusi Pecci, F. 1999, AJ, 118, 2839

Tosi, M., Ventura, P. \& D'Antona, F. 2001, in preparation

Vandenberg, D. A., Stetson, P. B., \& Bolte, M. 1996, ARA\&A, 34, 461

VandenBerg, D. A. 2000, ApJS, 129, 315 
Vazdekis, A., Salaris, M., Arimoto, N., \& Rose, J. A. 2001, ApJ, 549, 274

Ventura, P., Zeppieri, A., Mazzitelli, I., \& D'Antona, F. 1998, A\&A, 334, 953

\section{Discussion}

H. Lamers: The occurrence of red or blue horizontal branches is most likely related to the amount of envelope mass that remained on the stars after the Helium flash. Is it possible to relate the observed occurence of red or blue horizontal branches of globular clusters to any expected process that may have influenced the mass loss rate in the phases BEFORE the Helium flash?

F. D'Antona: Yes indeed. Your question raises again the problem of the 'second parameter' of Globular clusters. Mass loss is in fact the other important issue of "macrophysics" which we do not know how to manage from first principles. In GC stars, mass is probably lost not only at the helium flash, but also during the most luminous phases of the RGB evolution. The $\mathrm{H}$ layer left on the star at the HB phase depends of course also on the total mass which we see in evolution, and thus on the age, which, in principle, can affect the stars distribution in the HB. However, there are many cases in which we know that age is not the second parameter, and 'environmental' parameters affect the mass loss: e.g. the dynamical interactions among stars due to the stellar densities play a role, as nicely shown also here, in the poster by Catelan, Rood and Ferraro. The HB distribution of stars in M3 depends on the distance from the cluster center, the innermost cluster regions having many more blue HB stars than the outermost ones. This can only be explained if the stars in the denser regions have lost more mass on the RGB.

G. Harris: What are the possibile ways of changing the stellar models in order to reach the observed number ratio of clump stars versus red giant stars in NGC $1866 ?$

D'Antona: In the light of my talk, I stress that we can probably get a decent fit for the observed cluster, but this also means that theoretical models still do not have a satisfactory predictive power. In particular, our models for NGC 1866 reach a ratio of clump stars to $\mathrm{RGB}$ stars of $\mathrm{B} / \mathrm{R} \sim 0.7$, while the observed value is even larger $(\sim 0.9)$. I wished to stress that the clump is very dependent on the details of convection at several stages: on the one hand it depends on the maximum extension of the $\mathrm{H}$-envelope convection, which leaves a chemical $\mathrm{CNO}$ discontinuity crossed by the $\mathrm{H}$-burning shell during the core $\mathrm{He}$-burning stage, ultimately producing the loop. 'Non canonical' mixing mechanisms may also affect the chemical discontinuity. On the other hand, the ratio $\mathrm{B} / \mathrm{R}$ depends on the He-core burning stage (figure 4), which in part depends on the way we feed with fresh He the core. Instantaneous mixing and overshooting seems to give too small $\mathrm{B} / \mathrm{R}(\sim 0.3)$, as the blue loop occurs when the core He is already very small, but a precise tuning surely requires that we explore many other parameters in addition to the role of diffusive mixing (I wish to remember here, e.g., your examination of the role of metallicity, Harris and Deupree 1976). Paolo 
Ventura is preparing a discussion of this problem.

J.-H. Woo: You mentioned that the critical mass of core overshoot is $\sim 1.2 M_{\odot}$. How do you treat overshoot around the critical mass?

F. D'Antona: We do not "impose" a minimum mass for including overshooting. Our formulation is such that it fades away when the convective core mass disappears. So the reduction of the overshooting is autmatically taken into account in the models, and this is a more physical approach than assuming, say, a uniform $0.2 H_{p}$ of overshoot even when the core is barely $0.2 H_{p}$ in extension. Only detailed comparisons with obsevations will tell us if this schematization is helpful.

$L$. Vanzi: Since you said "the more metals, the worse the fits", we should be in great shape for the low metallicity systems. However, I am afraid that is NOT the case. So could you please tell us more about the problems of low metallicity?

F. D'Antona: For sure, the higher the metallicity, the worse is the problem. But also at low metallicity we still need to understand the absolute calibrations. E.g., our most recent stellar models for $[\mathrm{M} / \mathrm{H}]=-2$, taking into account nongrey FST boundary conditions, show that main sequence and red giants are affected by this choice of model atmospheres. And, in addition, even the color$T_{\text {eff }}$ relations from these new models computed by $\mathrm{F}$. Kupka and coworkers in Wien show differences of several $0.01 \mathrm{mag}$ in $B-V$ colors, with respect to standard MLT ATLAS9-based atmospheres. See Montalban et al. 2001, A\&A. 